\title{
Sportif Faaliyetlerde Kullanılmak Üzere Bir Uzman Sistem Tasarımı
}

\author{
Serkan Örücü ${ }^{1 *}$, Murat Selek ${ }^{2}$ \\ ${ }^{1}$ Karamanoğlu Mehmetbey Üniversitesi, Ermenek Meslek Yüksekokulu, Bilgisayar Teknolojileri Bölümü, Karaman, Türkiye (ORCID: 0000-0001-9905-2908) \\ ${ }^{2}$ Konya Teknik Üniversitesi, Teknik Bilimler Meslek Yüksekokulu, Elektronik ve Otomasyon Bölümü, Konya, Türkiye (ORCID: 0000-0001-8642-1823)
}

( $1^{\text {st }}$ International Conference on Computer, Electrical and Electronic Sciences ICCEES 2020 - 8-10 Ekim 2020)

(DOI: 10.31590/ejosat.802127)

ATIF/REFERENCE: Örücü, S. \& Selek, M. (2020). Sportif Faaliyetlerde Kullanılmak Üzere Bir Uzman Sistem Tasarımı. Avrupa Bilim ve Teknoloji Dergisi, (Özel Sayı), 176-183.

\section{$\ddot{O} \mathbf{z}$}

Vücut geliştirme gibi izotonik hareketlerin yapıldığı sporlarda ve rehabilitasyon süreçlerinde, antrenmanların eşzamanlı izlenmesi, yanlış yapılan hareketlerin anında düzeltilebilmesi antrenman yeterliliği ve veriminin belirlenebilmesi bireylerin yaralanma risklerinden uzak ve sağlıklı antrenman yapabilmeleri açısından hayati önem taşımaktadır. Çalışmamızda, bu amaca yönelik hareket modeline dayalı kural tabanlı bir Uzman Sistem (ES) tasarlanmıştır. Tasarlanan sistem, antrenör üzerinden elde edilen LR (Lateral Raise) antrenmanı verileri aracılığıyla, DTW metodu ile karşılaştırmalı olarak test edilmiştir. Test sonuçlarında, tasarladığımız ES'in aldığı kararların DTW metodundan elde edilen kararlar ile karşılaştırmasında \%64 oranında doğrulukla, daha yüksek oranda doğruluğa sahip olduğu görülmüştür.

Anahtar Kelimeler: Antrenman Doğruluğu, DTW, Hareket Modeli, İnsan-Makine Etkileşimi, Uzman Sistem.

\section{An Expert System Design for Use in Sports Activities}

\begin{abstract}
In sports and rehabilitation processes where isotonic movements such as bodybuilding are performed, it is vital to monitor the training simultaneously, correct the wrong movements, and determine the training adequacy and efficiency, so that individuals can do healthy training without risk of injury. For this purpose, in our study, a rule-based Expert System (ES) based on the motion model was designed. The designed system was tested comparatively with the DTW method via the LR (Lateral Raise) training data obtained from the coach. In the test results, it was seen that the decisions we made by the ES had a 64\% higher accuracy in comparison with the decisions obtained from the DTW method.
\end{abstract}

Keywords: DTW, Expert System, Human-Machine Interaction, Motion Model, Training Accuracy.

\section{Giriş}

Antrenman süreçlerinde yapılan hareketlerin eş zamanlı izlenmesi ve doğruluğunun tespiti biyomedikal, insan-makine etkileşimi, kinesyoloji, spor, rehabilitasyon vb. gibi çoğu bilim dalında dikkat çeken bir konudur. Bu süreçlerin eşzamanlı izlenmesi, sporcuların sağlıklı antrenman yapması için bir gerekliliktir. Böylece, harekete ilişkin motor beceriler gelişinceye kadar sporcunun yaralanma riski olmadan etkili antrenman yapması sağlanır (Appelbaum \& Erickson, 2018; Harris, Wilson, \& Vine, 2018). Bu amaçla çalışmamızda hareket modeline dayalı kural tabanlı bir uzman sistem tasarlanarak antrenman doğruluğunu belirlemede en çok kullanılan yöntemlerden olan DTW (Dinamik Zaman Bükme) yöntemiyle (Su, 2016), antrenör verileri üzerinden karşılaştırılmıştır.

\section{Materyal ve Metot}

${ }^{1}$ Serkan ÖRÜCÜ: Karamanoğlu Mehmetbey Üniversitesi, Ermenek Meslek Yüksekokulu, Bilgisayar Teknolojileri Bölümü, Karaman, Türkiye, ORCID: 0000-0001-9905-2908, srknorucu@kmu.edu.tr 


\subsection{Kinect V2 ile Eklem Açıları}

Çalışmalarımızda, saniyede 30 kare çekim yapabilen bir RGB kamera $(1920 \times 1080$ piksel $)$, bir derinlik sensörü $(512$ x 424 piksel ) ve bir mikrofondan oluşan Kinect V2 kullanılmıştır. Kinect V2 içerisinde yer alan derinlik sensörünün çalışması, karşısında bulunan nesneye IR (Kızılötesi) ışınlar gönderip cisimden yansıyan ışınların gitme ve yansıyıp geri gelme süresini ölçerek 3D derinliği algılama prensibine göre çalışır (Clark, Mentiplay, Hough, \& Pua, 2019; Konstantinidis, Bamparopoulos, \& Bamidis, 2017). 3D derinlik bilgisi, Kinect V2 tarafindan sunularak, kullanıcının eklem noktaların takip etmek amacıyla gerekli hesaplamaların yapılabilmesine imkân sağlar (Naeemabadi, Dinesen, Andersen, \& Hansen, 2019). Buradan elde edilen veriler, Şekil 1'de gösterildiği üzere eklemlerin 3D konumlarını vektörlerle ifade etmek için kullanılmıştır.

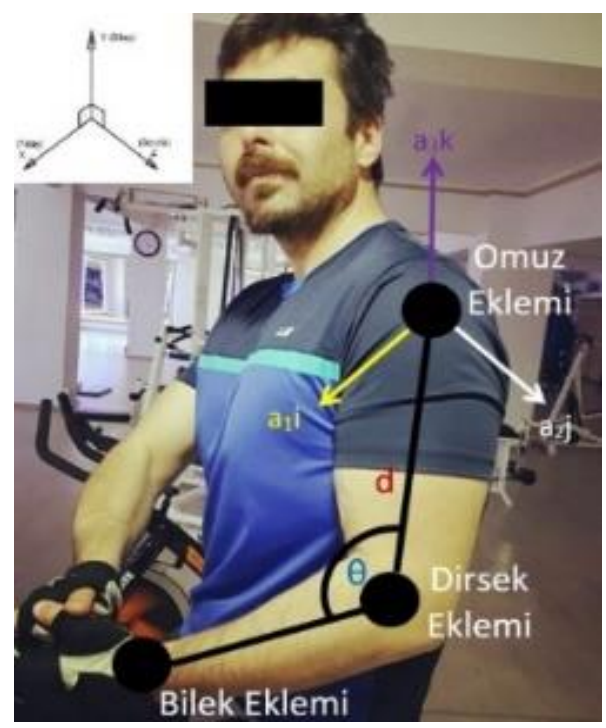

Şekil 1. Açılara dair örnek bir resim

Kinect V2'den elde edilen her bir derinlik verisi çerçevesinde bulunan herhangi bir pikselin derinliği üçgenleme prensibine göre üç boyutlu koordinatlara (1)'de gösterildiği gibi dönüştürülebilir. Bu sayede sensörden elde edilen bilgilerle, her hangi bir eklemin 3D koordinatının hesaplanabilmesine olanak sağlar.

$$
\begin{aligned}
& X=\frac{\left(x_{p}-p_{h} / 2\right)-\tan \left(\theta_{h} / 2\right)}{p_{h} / 2} * z_{p} \\
& Y=\frac{\left(p_{p}-y_{p}\right)-\tan \left(\theta_{v} / 2\right)}{p_{v} / 2} * z_{p} \\
& Z=z_{p}
\end{aligned}
$$

(1)'de "xp" derinlik görüntüsündeki bir pikselin yatay koordinatını, "yp" derinlik görüntüsündeki bir pikselin dikey koordinatını, "zp" bu koordinatlardaki pikselin derinlik değerini, "ph", yatay yönün toplam piksel sayısını, "pv", dikey yönün toplam piksel sayısını, " $\theta \mathrm{h}$ " IR kameranın yatay görüş açısını ve " $\theta \mathrm{v}$ " IR kameranın dikey görüş açısını ifade etmektedir. 3.1'e bağlı olarak eklemlerin R3'deki koordinatları hesaplandıktan sonra, eklemler için vektörler oluşturulmuş ve Dot product denilen çarpım işlemi gerçekleştirilmiştir.

Matematiksel olarak, Dot Product iki vektörü bir değer olarak alan ve sonuç olarak skaler değere döndüren süreçtir. Şekil 1'de gösterilen örnek üzerinden açıklarsak, el bileği - dirsek arasında oluşan sıfırdan farklı A ve dirsek - omuz arasında oluşan sıfırdan farklı B vektörleri $\vec{A}=\left\langle A_{1}, A_{2}, A_{3}, \ldots, A_{N}\right\rangle$ ve $\vec{B}=\left\langle B_{1}, B_{2}, B_{3}, \ldots, B_{N}\right\rangle$ olarak tanımlanan iki vektör olmak üzere Dot product (2)'de gösterilmiştir.

$$
A^{*} B=\sum_{i=1}^{n} A_{i} * B_{i}
$$

\subsection{Hareket Modeli Tasarımı}

Antrenman esnasında elde edilen verilerin işlenmesinde kullanılan yöntemler genellikle poz ve hareket benzerliği üzerine yoğunlaşmıştır. En doğru hareketi elde etmede uzman pozlarına başvurulmakta, ancak bunlarda da salon ortamındaki farklı ağırlıklarda, ya uzmanlar arasında hareket farklılıkları yâda bir kere yapılan hareketin bir daha birebir aynı yapılamaması durumu ortaya çıkmaktadır (Bailey, 2017). Bununla birlikte, antrenman hareketlerini yaparken hareket son formuna ulaşıncaya kadar olan süreçte sakatlanma yaşanabilmektedir (Reeves, Laskowski, \& Smith, 1998). Bu yüzden değerlendirmede kullanılan antrenman 
hareketlerini elde ederken dikkat edilmesi gereken esas husus, elde edilen poz ya da hareketin başlangıcından bitişine kadar olan sürecin sakatlıklara yol açmayacak şekilde modellenebilmesidir.

$\mathrm{Bu}$ amaca yönelik olarak, Beden Eğitimi ve Spor Yüksekokulu (Karamanoğlu Mehmetbey Üniversitesi) öğretim üyesi ve sertifikalı antrenör (Antalya Sport Center) görüşlerine başvurularak en çok yaralanmanın yaşandığı hareketlerden olan (Çetinkaya, Tanır, Atay, Bulut, \& Engin, 2017; Lavallee \& Balam, 2010; von Rottkay, Nöth, Zinner, \& Reichert, 2018) izotonik LR hareketi model alınarak hareket modeli tasarlanmıştır. Bu modelde, Şekil 2'de görülen hareket yapılırken omuz (A2 ve A3), dirsek (A1 ve A4), el bileği (A13 ve A14), kalça (A7 ve A8), diz (A9 ve A10), ayak bileği (A11 ve A12) ve omurga (A5) açılarındaki değişimler bir önceki kısımda açıklandığı gibi elde edilmektedir. Hareket modelinde, hareketin nasıl yapılması gerektiği ve hareket yanlış yapıldığında düzeltme amaçlı verilecek uyarılar da tanımlanmaktadır.

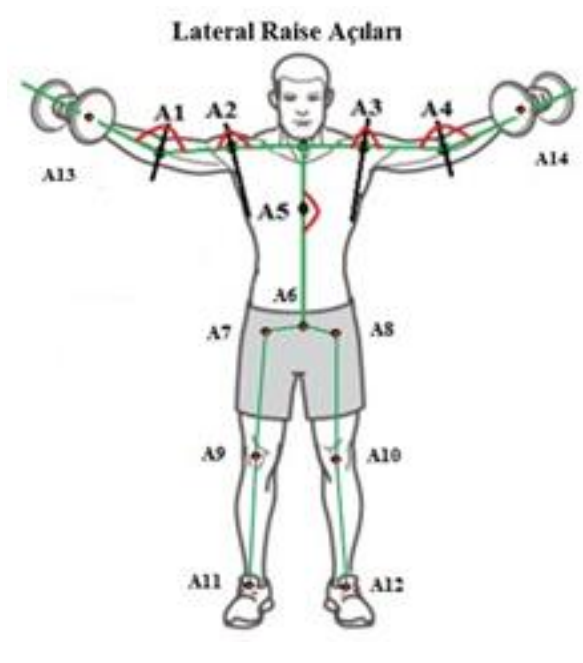

Şekil 2. Tasarlanan LR Hareket Modeli

LR antrenmanı için oluşturulan model, Şekil 2'de gösterildiği gibi açıklanırsa;

- Her elde bir dambıl ile $170^{\circ}-180^{\circ}$ aralığında (A5) dik durulmalı (3) (Taniguchi, 1997),

$R_{1}=\left\{\begin{array}{c}1,170 \leq \mathrm{A} 5<180 \\ 0, \text { Otherwise }\end{array}\right.$

- Ayaklar arası mesafe (A11-A12) omuz genişliğinden (A2-A3) biraz daha dar ve kalça (A7-A8) genişliğinden biraz daha fazla olmalı (4) (Arandjelović, 2013; Taniguchi, 1997),

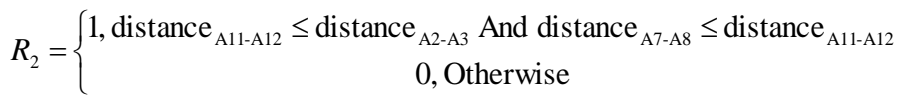

- Kollar, dirseklerde (A1 ve A4) $140^{\circ}-160^{\circ}$ aralığında açılmalı (5) (Andersen, Vinstrup, Jakobsen, \& Sundstrup, 2017),

$R_{3}=\left\{\begin{array}{c}1,140 \leq \mathrm{A} 1 \leq 160 \text { and } 140 \leq \mathrm{A} 4 \leq 160 \\ \text { 0, Otherwise }\end{array}\right.$

- Dirsekler (A1 ve A4) omuz hizasında (A2 ve A3) $15^{\circ}-30^{\circ}$ aralığında açı oluşturuncaya kadar kollar yanlara doğru kaldırılmalı (6) (Andersen et al., 2017; Taniguchi, 1997),

$R_{4}=\left\{\begin{array}{c}1,15 \leq \theta_{A 1-A 2} \leq 30 \text { and } 15 \leq \theta_{A 3-A 4} \leq 30 \\ 0, \text { Otherwise }\end{array}\right.$

- Hareketin en son noktasında, bilekler (A13 ve A14) dirsekler (A1 ve A4) ile $10^{\circ}-30^{\circ}$ yapacak kadar yukarıda olmalı (7) (Andersen et al., 2017; Keogh, Aickin, \& Oldham, 2010), 
$R_{5}=\left\{\begin{array}{c}1,10 \leq \theta_{A 1-A 13} \leq 30 \text { and } 10 \leq \theta_{A 4-A 14} \leq 30 \\ \text { O, Otherwise }\end{array}\right.$

- Hareket doğru yapıldığında ekranda kullanıcıya "Doğru Hareket" mesajı verilmekte, yanlış yapıldığında ise Tablo 1 'de gösterilen mesajlar geri dönüt olarak verilmektedir.

Tablo 1. LR Modeli için Kullanıcıya Verilen Eşzamanlı Uyarılar

\begin{tabular}{cc}
\hline Uyarı No. & Uyarılar \\
\hline 1 & Lütfen Dik Durunuz ve Ayaklarınızı Uygun Konuma Alınız \\
\hline 2 & Ağırlığı Çok Kaldırmayınız \\
\hline 3 & Dirsek Açıları Yetersiz, Dirseğinizi Daha Çok Açınız \\
\hline 4 & Omuz Açıları Yetersiz, Ağırlığı Daha Çok Kaldırınız \\
\hline 5 & Omuz ve Dirsek Açılarınız Hatalı, Sakatlanma Riski Var, Lütfen Daha Hafif Ağırlık \\
& Seçin. \\
\hline 6 & Ağırlığı Daha Çok Kaldırınız. \\
\hline 7 & Doğru Hareket \\
\hline 8 & Hareketiniz Yanlış. Lütfen Antrenörünüzle Görüşün, Antrenman Sonlandırılıyor. \\
\hline
\end{tabular}

\subsection{Uzman Sistem Tasarımı}

Uzman Sistem, belirli alanlarda karşılaşılan bir veya daha fazla bireysel yargı yetisi ve karar alma sürecinin kopyalanmasıyla oluşturulan karar destek yazılımıdır (Selek, Başçiftçi, \& Örücü, 2017). Bu program tarafından kullanılan bilgi ve mantıksal çıkarım mekanizması, alanında uzman kişi ya da kişilerin bilgi ve mantıksal çıkarım mekanizmasına göre modellenmektedir (Wagner, 2017). Ayrıca, modelleme sürecinde kullanılacak karar yapılarının tartışmasız doğruluğa sahip olması gerekir. Böylelikle, kullanıcılara ihtiyaçları ve istekleri doğrultusunda bir ES ile rehberlik edilebilir. Uzman Sistemlerin en önemli bölümlerinden biri olan bilgi veri tabanı, alanında uzman kişi veya kişilerin bilgi dağarcığına göre karar kuralları olarak oluşturulup güncellenmektedir (Başçiftçi \& Avuçlu, 2018). Bu kuralları oluştururken "If - Then" yapıları kullanılır. Bu yapılar şu şekilde ifade edilir;

\section{If (one or more condition $=$ True $)$ then $($ outcome/result $)$}

Bu yapı oluşturulurken birden fazla koşul kullanılabilir. Bu koşullar, duruma bağlı olarak “And” ve "Or” ifadeleri kullanılarak bağlanmaktadır. Örneğin, iki koşul kontrol ediliyorsa, "And” ifadesi kullanılarak koşulların her ikisinin de doğrulanması gerçekleşir. Ancak iki koşuldan sadece biri yeterliyse "Or” ifadesi kullanılır. Spor alanına ilişkin bir örnek şu şekilde ifade edilebilir;

\section{Algoritma 1. Spor Alanına İlişkin Örnek Bir Algoritma}

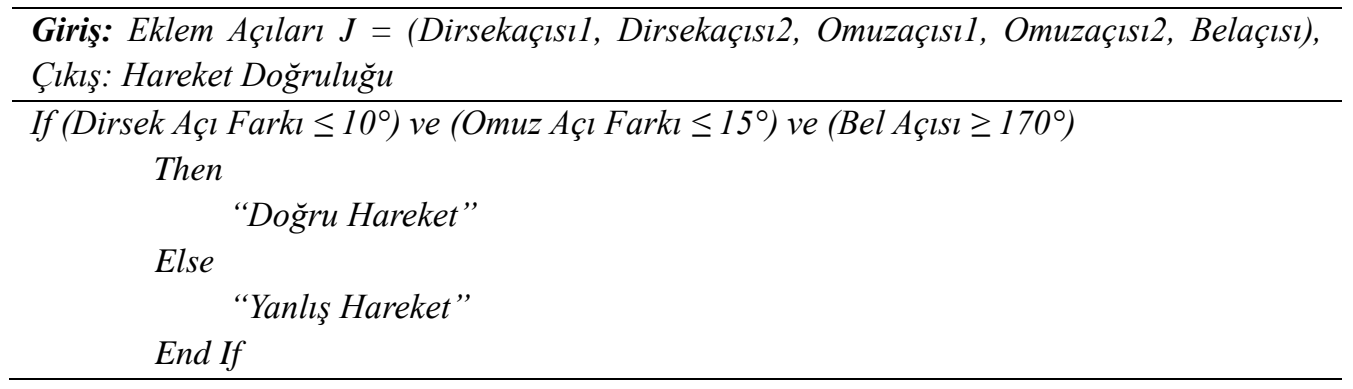

$\mathrm{Bu}$ amaca uygun tasarlanan LR hareket modeli çalışmadaki ES içinde kural tabanı olarak kullanılmış, hareket açılarının değişimine göre 8 kural (R1 - R8) ve 256 koşuldan oluşan bir doğruluk tablosu tasarlanarak Tablo 2'de örnek bir tanesi sunulmuştur.

Tablo 2. Örnek Bir Koşulun Çıkış Değerleri

\begin{tabular}{cccccccccc}
\hline \multirow{2}{*}{ Y Verilen uyarı } & \multicolumn{1}{l}{ Kural Koşulları } & & & & \\
& & Q1 & Q2 & Q3 & Q4 & Q5 & Q6 & Q7 & Q8 \\
\hline 1 & 5 & $\mathrm{x}$ & $\mathrm{x}$ & 1 & 1 & $\mathrm{x}$ & 1 & $\mathrm{x}$ & $\mathrm{x}$ \\
\hline
\end{tabular}


Bu tabloda, örnek bir kural sistem tarafindan verilen uyarılar (Y), verilen uyarı (Bkz. Tablo 1) ve kural durumlarıyla (Q1-Q8) birlikte gösterilmiştir.

\subsection{Dinamik Zaman Bükme}

Zaman serilerinde, Dinamik Zaman Bükme (Dynamic Time Warp - DTW) metodu zaman ya da hız bakımından farklılık gösteren iki sinyalin benzerlik ölçümü için kullanılan bir metottur (Varatharajan, Manogaran, Priyan, \& Sundarasekar, 2018). Örneğin; farklı kişilerin aynı antrenmanı yapma hızları farklı olabilir ya da antrenmanın kasılma ve gevşeme evrelerinde ki hızlanmalar veya yavaşlamalar farklı olabilir.

DTW yöntemi aracılığıyla, Antrenörün ve sporcunun antrenman verilerine ait benzerlik oranları, bu iki veri serisi arasındaki zamansal kaymaların ortadan kaldırılmasıyla hesaplanabilir. Şekil 3'de LR antrenmanının 1 tekrarında elde edilen omuz açılarındaki değişim Antrenör (m) ve Sporcu (a) olarak gösterilmiştir.

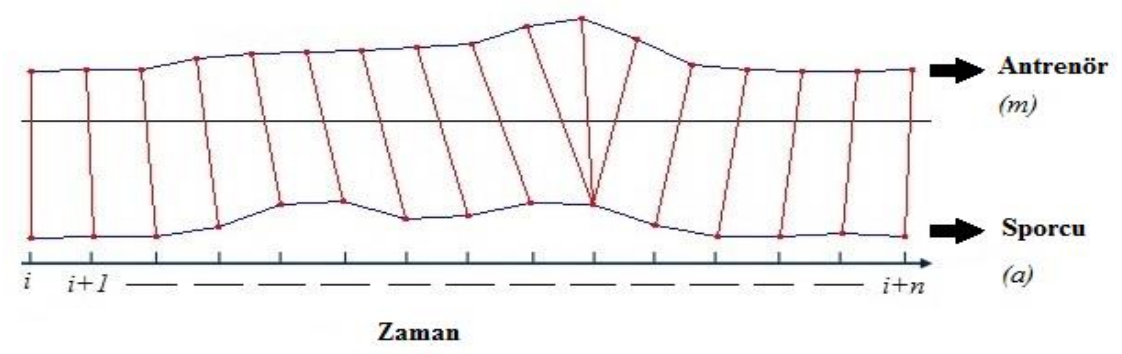

Şekil 3. LR Antrenmanının 1 Tekrarında Elde Edilen Omuz Açıları

DTW metodu kullanılarak bu verilerin benzerliği bulunmak istenirse, sirasıyla $M=m 1, m 2, \ldots ., m N$ ve $A=a 1, a 2, \ldots ., a X$ olarak ifade edilerek, dinamik programlama aracılığıyla $\mathrm{N}^{*} \mathrm{X}$ boyutunda bir uzaklık matrisi oluşturulur (Brill et al., 2019). Bu matrisin her ( $\left.n, x\right)$ elemanı $M N$ ve $A X$ arasındaki genlik bakımından uzaklık olan $d(m n, a x)$ 'i içerir (8).

$d\left(m_{n}, a_{x}\right)=\sqrt{\left|m_{n}^{2}-a_{x}^{2}\right|}$

Bu işlem için en çok kullanılan fonksiyonlar ise (9) ve (10)'da verilen öklid ve farkın karesi fonksiyonlarıdır (Brill et al., 2019; Morel, Achard, Kulpa, \& Dubuisson, 2018).

$d(m, a)=\left|m_{n}-a_{x}\right|$
$d(m, a)=\left(m_{n}-a_{x}\right)^{2}$

Böylece, toplam uzaklığı minimize ederek, $(1,1)$ elemanından başlayıp (N,A)'da biten ve her bir adımda gidilecek en kısa yolların birleşiminden oluşan bükme yolu (11)'de gösterildiği gibi bulunur.

$W=w_{1}, w_{2}, \ldots, w_{K}$

Matematiksel olarak, uzaklık matrisinin elemanları d(n,a), ilk satırın ilk n elemanından her hangi birinden başlayan ve (n,a)'inci elemana kadar gelen minimum uzaklık yolundaki toplam uzaklığı saklamaktadır. $d(n, a)$ değeri $n=2, \ldots, N$ ve $a=2, \ldots, X$ için $(12)$ 'de gösterildiği gibi hesaplanır. Burada, C fonksiyonu (n,x) noktasının, ideal bükme yolu eğimine göre en uygun hizalama ile ilerlemesi gerektiğini hesaplar ve bükme yolu uzunluğunu da kontrol eder (Brill et al., 2019).

$$
D(n, x)=d\left(M_{n}, A_{x}\right)+\min \left\{\begin{array}{c}
D(n-1, x-1)+C(n-1, v-1), \\
D(n-1, x)+C(n-1, x), \\
D(n, x-1)+C(n, x-1)
\end{array}\right.
$$

Şekil 4'de gösterildiği üzere, en kısa yol (m1,a1) noktasından başlanarak (mn,ax) noktasına ulaşana kadar ilerlenerek hesaplanır. 


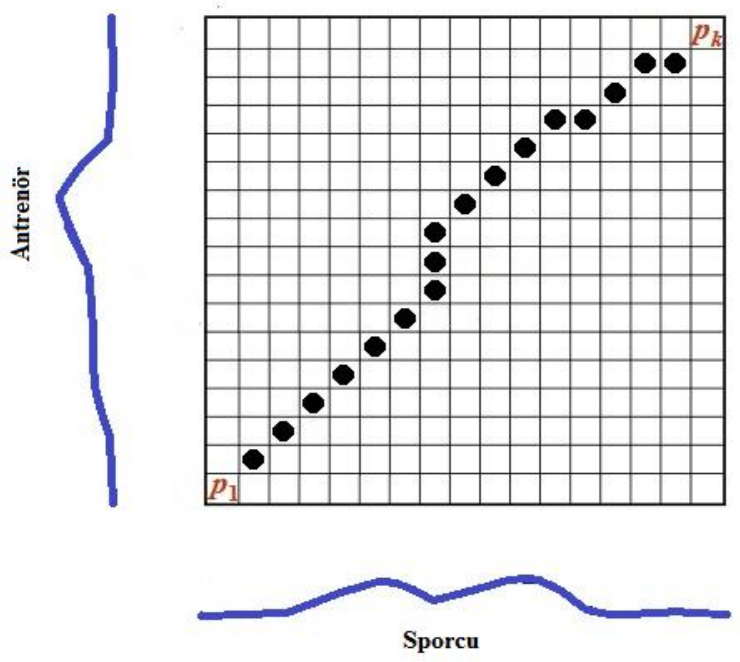

Şekil 4. DTW'de En Klsa Yol Hesaplama

\section{Araştırma Sonuçları ve Tartışma}

Antrenörden 1 tekrar (\%25-30 ağırlıkla) LR hareketleri gerçekleştirmesi istenmiştir. Bu hareketlerden elde edilen Kinect V2 verileri Şekil 5'de gösterildiği üzere DTW yöntemi için model olarak kullanılmıştır.

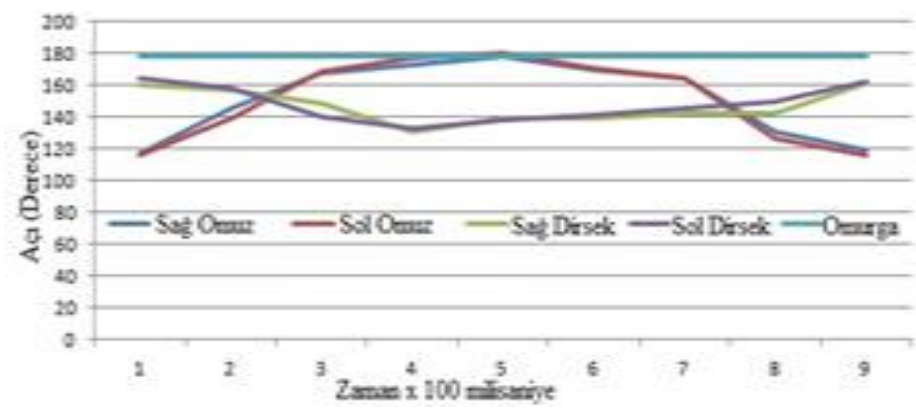

Şekil 5. Model LR Antrenmanı Verileri

Çalışmamızda, salon ortamında ağırlıklarla yapılan izotonik hareketlerde farklı ağırlıklarda bile hareket farklılıkları oluşabildiğinden, antrenörden LR antrenmanı yapması istenmiştir (Adelsberger \& Tröster, 2013; Ericsson, 2017; Jeong et al., 2016). 3 set 10 tekrar olarak yapılan bu antrenmanlarda, antrenör ilk seti \%30-40, ikinci seti \% 50-70 ve son seti \%80-100 oranında ağırlık kullanarak (17.5 kg. max.) yapmıştır (Mateo et al., 2018; Yu \& Xiong, 2019).

Buradan elde edilen veriler DTW ve modele göre tasarlanan kural tabanlı ES ile işlenerek karşılaştırılmış ve Şekil 6'da gösterilmiştir.

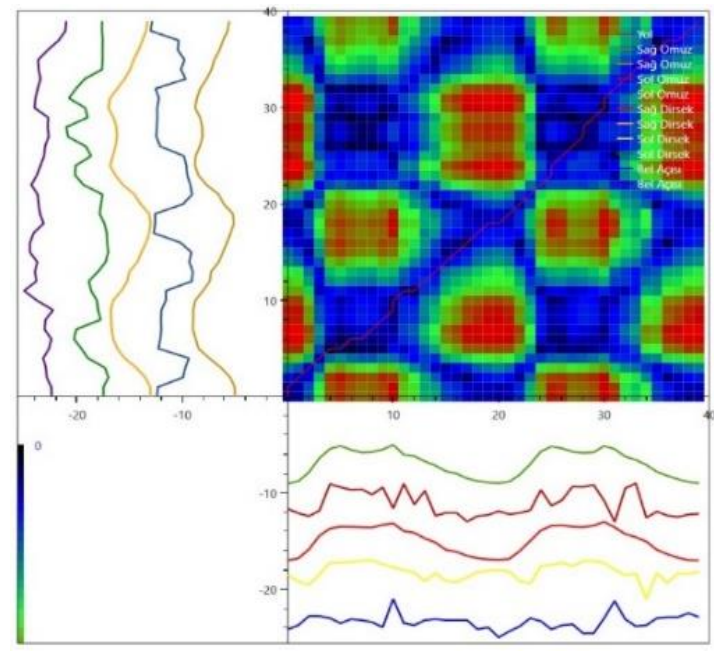

Şekil 6. DTW metodu ile LR antrenmanı analizi 
Öncelikle, DTW metodundan elde edilen veriler Şekil 7'de gösterilmiştir.

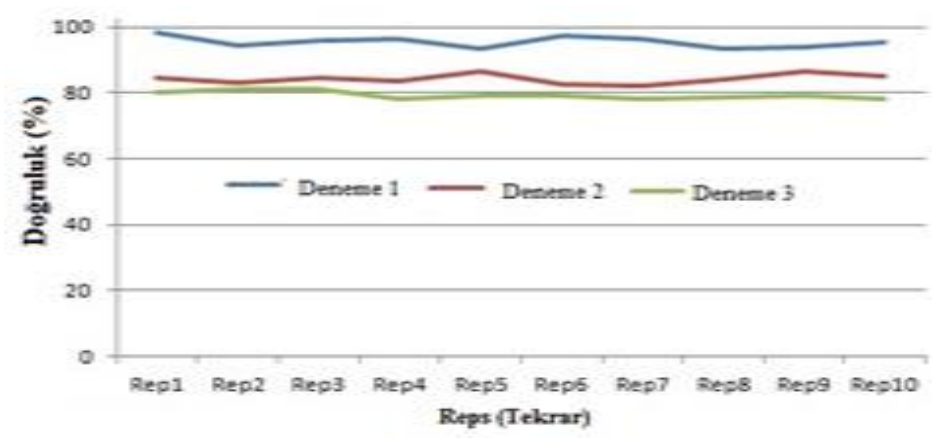

Şekil 7. DTW Metodundan Elde Edilen LR Antrenmanı Doğruluk Oranları

Şekil 7'de görüldüğü üzere antrenmanlarda ağılık arttırıldıkça hareketteki bozulma oranı artmakta ve doğruluk oranı da düşmektedir. Bu noktada, DTW'nin antrenmanlarda kullanılabilirlik seviyesini belirlemek için LR antrenmanlarından elde edilen verilerin Ortalama ve Standart Sapma değerleri hesaplanmıştır. Bu hesaplama sonucu antrenmanlarda kullanılacak doğruluk oranı LR için \%86.43 \pm 7.02 olarak tespit edilmiştir.

$\mathrm{Bu}$ orana göre veriler yeniden işlendiğinde, DTW'nin LR hareketinde \%56 oranında hareket doğruluğunu tespit edebildiği görülmektedir. Aynı veriler ES ile işlenerek elde edilen veriler antrenman türü, deneme numarası, hareket doğruluğu ve sistem uyarıları (Bkz Tablo 1) ile birlikte Tablo 3'de gösterilmiştir.

Tablo 3. LR Hareketli için Hareket Doğruluğu ve Sistem Uyarları

\begin{tabular}{|c|c|c|c|c|c|c|c|c|c|c|c|}
\hline \multirow[b]{2}{*}{ Tekrar No. } & \multirow[b]{2}{*}{ Karar Türü } & \multicolumn{10}{|c|}{ Tekrarlar } \\
\hline & & 1 & 2 & 3 & 4 & 5 & 6 & 7 & 8 & 9 & 11 \\
\hline \multirow[b]{2}{*}{1} & Doğruluk & $\sqrt{ }$ & $\mathrm{X}$ & $\sqrt{ }$ & $\sqrt{ }$ & $X$ & $\sqrt{ }$ & $\sqrt{ }$ & $X$ & $\sqrt{ }$ & $\sqrt{ }$ \\
\hline & Uyart & 7 & 1 & 7 & 7 & 8 & 7 & 7 & 3 & 7 & 7 \\
\hline \multirow[b]{2}{*}{2} & Doğruluk & $X$ & $\sqrt{ }$ & $X$ & $X$ & $X$ & $\sqrt{ }$ & $\sqrt{ }$ & $X$ & $X$ & $X$ \\
\hline & Uyarl & 3 & 7 & 5 & 5 & 3 & 7 & 7 & 4 & 1 & 1 \\
\hline \multirow[b]{2}{*}{3} & Doğruluk & $X$ & $\mathrm{X}$ & $\mathrm{X}$ & $X$ & $X$ & $\mathrm{X}$ & $\mathrm{X}$ & $X$ & $X$ & $\mathrm{X}$ \\
\hline & Uyarl & 4 & 1 & 1 & 3 & 5 & 4 & 4 & 5 & 1 & 1 \\
\hline
\end{tabular}

Şekil 7 ve Tablo 3 verileri incelenirse, ES'in hareket doğruluğunu LR hareketinde \%64 oranında tespit edebildiği görülmüştür. Ayrıca, tasarladığımız ES'in hem hareket doğruluğunu DTW modelinden daha yüksek oranda belirleyebildiği hem de hareketteki bozulmaları gösterip bunları düzeltmeye yönelik verileri kullanıcılara sunabildiği görülmektedir.

\section{Sonuç}

Tasarladığımız ES'in aldığı kararlar DTW metodundan elde edilen sonuçlardan daha yüksek oranda doğruluğa sahip olması, sistemin spor alanında (Ojeda-Castelo, Piedra-Fernandez, Iribarne, \& Bernal-Bravo, 2018) ve rehabilitasyon alanında (Rybarczyk, Kleine Deters, Cointe, \& Esparza, 2018), ev - spor salonu ortamında kullanıma uygun olduğunu işaret etmektedir.

\section{Teşekkür}

Hareket modelinin oluşturulmasında verdikleri destekler için Dr. Öğr. Üyesi Yusuf ER ve Uzman Antrenör Atilla SÖNMEZIŞIK'a teşekkür ederiz.

\section{Kaynakça}

Adelsberger, R., \& Tröster, G. (2013, 6-9 May 2013). Experts lift differently: Classification of weight-lifting athletes. Paper presented at the 2013 IEEE International Conference on Body Sensor Networks.

Andersen, L. L., Vinstrup, J., Jakobsen, M. D., \& Sundstrup, E. (2017). Validity and reliability of elastic resistance bands for measuring shoulder muscle strength. Scandinavian Journal of Medicine \& Science in Sports, 27(8), 887-894. doi:10.1111/sms. 12695

Appelbaum, L. G., \& Erickson, G. (2018). Sports vision training: A review of the state-of-the-art in digital training techniques. International Review of Sport and Exercise Psychology, 11(1), 160-189. doi:10.1080/1750984X.2016.1266376 
Arandjelović, O. (2013). Does cheating pay: the role of externally supplied momentum on muscular force in resistance exercise. European Journal of Applied Physiology, 113(1), 135-145. doi:10.1007/s00421-012-2420-y

Bailey, R. (2017). Sport, physical activity and educational achievement - towards an explanatory model. Sport in Society, 20(7), 768788. doi:10.1080/17430437.2016.1207756

Başçiftçi, F., \& Avuçlu, E. (2018). An expert system design to diagnose cancer by using a new method reduced rule base. Computer Methods and Programs in Biomedicine, 157, 113-120. doi:https://doi.org/10.1016/j.cmpb.2018.01.020

Brill, M., Fluschnik, T., Froese, V., Jain, B., Niedermeier, R., \& Schultz, D. (2019). Exact mean computation in dynamic time warping spaces. Data Mining and Knowledge Discovery, 33(1), 252-291. doi:10.1007/s10618-018-0604-8

Clark, R. A., Mentiplay, B. F., Hough, E., \& Pua, Y. H. (2019). Three-dimensional cameras and skeleton pose tracking for physical function assessment: A review of uses, validity, current developments and Kinect alternatives. Gait \& Posture, 68, 193-200. doi:https://doi.org/10.1016/j.gaitpost.2018.11.029

Çetinkaya, E., Tanır, H., Atay, E., Bulut, Ç., \& Engin, H. (2017). Investigation of musculoskeletal system injuries in athletes doing bodybuilding and fitness sports\&lt;p\&gt;Vücut geliştirme ve fitness sporu yapanlarda, kas, iskelet sistemi sakatlıklarının belirlenmesi. Journal of Human Sciences, 14(4), 4023-4031.

Ericsson, K. A. (2017). Expertise and individual differences: the search for the structure and acquisition of experts' superior performance. Wiley Interdisciplinary Reviews: Cognitive Science, 8(1-2), e1382. doi:10.1002/wcs.1382

Harris, D. J., Wilson, M. R., \& Vine, S. J. (2018). A Systematic Review of Commercial Cognitive Training Devices: Implications for Use in Sport. Front. Psychol., 9(709). doi:10.3389/fpsyg.2018.00709

Jeong, H., Yamada, K., Kido, M., Okada, S., Nomura, T., \& Ohno, Y. (2016). Analysis of Difference in Center-of-Pressure Positions Between Experts and Novices During Asymmetric Lifting. IEEE Journal of Translational Engineering in Health and Medicine, 4, 1-11. doi:10.1109/JTEHM.2016.2599185

Keogh, J. W. L., Aickin, S. E., \& Oldham, A. R. H. (2010). Can Common Measures of Core Stability Distinguish Performance in a Shoulder Pressing Task Under Stable and Unstable Conditions? The Journal of Strength \& Conditioning Research, 24(2), 422429. doi:10.1519/JSC.0b013e3181c7c6b9

Konstantinidis, E. I., Bamparopoulos, G., \& Bamidis, P. D. (2017). Moving Real Exergaming Engines on the Web: The webFitForAll Case Study in an Active and Healthy Ageing Living Lab Environment. IEEE Journal of Biomedical and Health Informatics, 21(3), 859-866. doi:10.1109/JBHI.2016.2559787

Lavallee, M. E., \& Balam, T. (2010). An Overview of Strength Training Injuries: Acute and Chronic. Current Sports Medicine Reports, 9(5), 307-313. doi:10.1249/JSR.0b013e3181f3ed6d

Mateo, F., Soria-Olivas, E., Carrasco, J. J., Bonanad, S., Querol, F., \& Pérez-Alenda, S. (2018). HemoKinect: A Microsoft Kinect V2 Based Exergaming Software to Supervise Physical Exercise of Patients with Hemophilia. Sensors, 18(8), 2439.

Morel, M., Achard, C., Kulpa, R., \& Dubuisson, S. (2018). Time-series averaging using constrained dynamic time warping with tolerance. Pattern Recognition, 74, 77-89. doi:https://doi.org/10.1016/j.patcog.2017.08.015

Naeemabadi, M., Dinesen, B., Andersen, O. K., \& Hansen, J. (2019). Influence of a Marker-Based Motion Capture System on the Performance of Microsoft Kinect v2 Skeleton Algorithm. IEEE Sensors Journal, 19(1), 171-179. doi:10.1109/JSEN.2018.2876624

Ojeda-Castelo, J. J., Piedra-Fernandez, J. A., Iribarne, L., \& Bernal-Bravo, C. (2018). KiNEEt: application for learning and rehabilitation in special educational needs. Multimedia Tools and Applications, 77(18), 24013-24039. doi:10.1007/s11042-018$5678-1$

Reeves, R. K., Laskowski, E. R., \& Smith, J. (1998). Weight Training Injuries. The Physician and Sportsmedicine, 26(3), 54-73. doi:10.1080/00913847.1998.11440348

Rybarczyk, Y., Kleine Deters, J., Cointe, C., \& Esparza, D. (2018). Smart Web-Based Platform to Support Physical Rehabilitation. Sensors, 18(5), 1344.

Selek, M., Başçiftçi, F., \& Örücü, S. (2017). Designing medical expert system based on logical reduced rule for basic malaria diagnosis from malaria signs and symptoms. World Journal of Engineering, 14(3), 227-230. doi:10.1108/WJE-10-2016-0112

$\mathrm{Su}, \mathrm{C} . \mathrm{H}$. (2016). Developing and evaluating effectiveness of 3D game-based rehabilitation system for Total Knee Replacement Rehabilitation patients. Multimedia Tools and Applications, 75(16), 10037-10057. doi:10.1007/s11042-015-2820-1

Taniguchi, Y. (1997). Lateral specificity in resistance training: the effect of bilateral and unilateral training. European Journal of Applied Physiology and Occupational Physiology, 75(2), 144-150. doi:10.1007/s004210050139

Varatharajan, R., Manogaran, G., Priyan, M. K., \& Sundarasekar, R. (2018). Wearable sensor devices for early detection of Alzheimer disease using dynamic time warping algorithm. Cluster Computing, 21(1), 681-690. doi:10.1007/s10586-017-0977-2

von Rottkay, E., Nöth, U., Zinner, J., \& Reichert, J. C. (2018). Schulterverletzungen im CrossFit und verwandten Sportarten. Sports Orthopaedics and Traumatology, 34(2), 145-150. doi:https://doi.org/10.1016/j.orthtr.2017.12.007

Wagner, W. P. (2017). Trends in expert system development: A longitudinal content analysis of over thirty years of expert system case studies. Expert Systems with Applications, 76, 85-96. doi:https://doi.org/10.1016/j.eswa.2017.01.028

Yu, X., \& Xiong, S. (2019). A Dynamic Time Warping Based Algorithm to Evaluate Kinect-Enabled Home-Based Physical Rehabilitation Exercises for Older People. Sensors, 19(13), 2882. 